International Journal of Pure and Applied Mathematics

Volume 100 No. 2 2015, 309-335

ISSN: 1311-8080 (printed version); ISSN: 1314-3395 (on-line version)

url: http://www.ijpam.eu

doi: http://dx.doi.org/10.12732/ijpam.v100i2.11

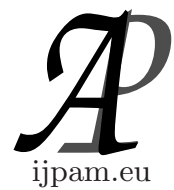

\title{
A NEW EXTENDED GAMMA GENERALIZED MODEL
}

\author{
Ronaldo V. da Silva ${ }^{1}$, Frank Gomes-Silva ${ }^{2}$, \\ Manoel Wallace A. Ramos ${ }^{3}$, Gauss M. Cordeiro ${ }^{4}$ \\ ${ }^{1}$ Recife Military School \\ Recife, 52070-080, BRAZIL \\ ${ }^{2}$ Department of Statistics and Informatics \\ Federal Rural University of Pernambuco \\ Recife, 52171-900, BRAZIL \\ ${ }^{3}$ Department of Mathematics \\ Federal Institute of Paraíba \\ João Pessoa, 58015-020, BRAZIL \\ ${ }^{4}$ Department of Statistics \\ Federal University of Pernambuco \\ Recife, 50740-540, BRAZIL
}

\begin{abstract}
We propose a new five-parameter extended gamma generalized model. Some special cases are discussed. We derive some mathematical properties of the proposed distribution including explicit expressions for the quantile function, ordinary and incomplete moments, generating function, mean deviations, reliability, order statistics and their moments and Rényi entropy. The method of maximum likelihood is used to estimate the model parameter. We ilustrate the potentiality of the new model with an application to a real data set.
\end{abstract}

AMS Subject Classification: 60E05

Key Words: beta generalized, gamma distribution, exponentiated gamma distribution, exponentiated generalized gamma distribution, maximum likelihood estimation, moment

Received: January 4, 2015

(C) 2015 Academic Publications, Ltd.

${ }^{\S}$ Correspondence author url: www.acadpubl.eu 


\section{Introduction}

For a continuous baseline cumulative distribution function (cdf) $G(x)$, Cordeiro et al. [3] defined the exponentiated generalized ("EG" for short) class by

$$
F(x ; a, b)=\left\{1-[1-G(x)]^{a}\right\}^{b}, \quad x \in D \subset \mathbb{R}
$$

where $a>0$ and $b>0$ are two additional parameters whose role is to introduce skewness and to vary tail weights. Because of its tractable distribution function (1), the EG class can be used quite effectively even if the data are censored. Equation (1) is a continuous univariate family of distributions for modeling univariate data that can be in any interval of the real line. Therefore, this family is motivated to analyze continuous univariate data that have any type of support. Further, this family allows for greater flexibility of its tails and can be widely applied in many areas of engineering and biology. Correspondingly, its probability density function (pdf) has a very simple form

$$
f(x ; a, b)=a b g(x)[1-G(x)]^{a-1}\left\{1-[1-G(x)]^{a}\right\}^{b-1} .
$$

One major benefit of the density (2) is its ability of fitting skewed data that can not be properly fitted by existing distributions. Based on the cdf $G(x)$ and pdf $g(x)$ of any baseline $\mathrm{G}$ distribution, we can associate the EG-G density function (2) with two extra shape parameters $a$ and $b$. The role of these parameters is to govern skewness and generate distributions with heavier/ligther tails. The two additional parameters induced by the EG generator are sought as a manner to furnish a more flexible distribution. The EG-G family has a wide variety of shapes and it is able to model comfortable bathtub-shaped failure rate data. Further, it can easily used for discriminating between the $\mathrm{G}$ and EG-G distributions.

The baseline distribution $G(x)$ is a special case of (1) when $a=b=1$. Setting $a=1$ gives the exponentiated-G (exp-G) distribution. So, the distribution given by (2) generalizes both exponentiated Lehmman type alternative distributions. Note that even if $g(x)$ is a symmetric distribution, the distribution $f(x)$ will not be a symmetric distribution.

The class of exponentiated generalized distributions shares an attractive physical interpretation whenever $a$ and $b$ are positive integers. Consider a device made of $b$ independent components in a parallel system. Furthermore, each component is made of $a$ independent subcomponents identically distributed according to $G(x)$ in a series system. The device fails if all $b$ components fail and each component fails if any subcomponent fails. Let $X_{j 1}, \ldots, X_{j a}$ denote 
the lifetimes of the subcomponents within the $j$ th component, $j=1, \ldots, b$, with commom cdf $G(x)$. Let $X_{j}$ denote the lifetime of the $j$ th component and let $X$ denote the lifetime of the device. Thus the cdf of $X$ is

$$
\begin{aligned}
P(X \leq x) & =P\left(X_{1} \leq x, \ldots, X_{b} \leq x\right)=P\left(X_{1} \leq x\right)^{b} \\
& =\left[1-P\left(X_{1}>x\right)\right]^{b}=\left[1-P\left(X_{11}>x, \ldots, X_{1 a}>x\right)\right]^{b} \\
& =\left\{1-\left[P\left(X_{11}>x\right)\right]^{a}\right\}^{b}=\left\{1-\left[1-P\left(X_{11} \leq x\right)\right]^{a}\right\}^{b} .
\end{aligned}
$$

So, it follows that the lifetime of the device obeys the exponentiated generalized type distribution.

The hazard rate function (hrf) corresponding to (2) is given by

$$
\tau(x ; a, b)=\frac{a b g(x)[1-G(x)]^{a-1}\left\{1-[1-G(x)]^{a}\right\}^{b-1}}{1-\left\{1-[1-G(x)]^{a}\right\}^{b}} .
$$

The most general form of the gamma distribution is the three parameter generalized gamma (GG) distribution studied by Stacy [16]. It includes as special models the exponential, Weibull, gamma, Rayleigh, among other models. This distribution is suitable for modeling data with different types of hrf: increasing, decreasing, bathtub shaped and unimodal, which makes it particularly useful for estimating individual hazard functions. The GG distribution has been used in several research areas such as engineering, hydrology and survival analysis. Its probability density function (pdf) is given by

$$
g(x ; c, \beta, \lambda)=\frac{c \lambda^{c \beta}}{\Gamma(\beta)} x^{c \beta-1} \mathrm{e}^{-(\lambda \mathrm{x})^{c}}, \mathrm{x}>0,
$$

where $\lambda>0$ is a scale parameter, $\beta>0$ and $c>0$ are shape parameters and $\Gamma(\beta)=\int_{0}^{\infty} \omega^{\beta-1} \mathrm{e}^{-\omega} \mathrm{d} \omega$ is the gamma function. The Weibull, gamma and halfnormal distributions correspond to $\beta=1, c=1$ and $\beta=3, c=2$, respectively. In addition, the log-normal distribution is a limiting special case when $\beta \rightarrow \infty$.

The cdf corresponding to (4) is

$$
G(x ; c, \beta, \lambda)=\frac{\gamma\left(\beta,(\lambda x)^{c}\right)}{\Gamma(\beta)},
$$

where $\gamma(\beta, x)=\int_{0}^{x} \omega^{\beta-1} \mathrm{e}^{-\omega} \mathrm{d} \omega$ is the incomplete gamma function.

Gupta et al. [6] first proposed a generalization of the standard exponential distribution. The exponentiated exponential (EE) distribution is defined by the cdf

$$
F(x ; a, \lambda)=\left(1-e^{-\lambda x}\right)^{\alpha}
$$


for $x>0, \lambda>0$ and $\alpha>0$, which is simply the $\alpha$ th power of the cdf of the standard exponential distribution. For a discussion and some mathematical properties, see Gupta and Kundu [7].

Nadarajah and Kotz [11] introduced the exponentiated gamma (EГ) distribution which is obtained in the same way as the EE distribution. They also introduced two new distributions, the exponentiated Fréchet (EF) and the exponentiated Gumbel (EGu), although the way they defined the cdf for these two distributions is slightly different. For instance, the cdf of the EGu distribution is defined by

$$
F(x ; \alpha, \mu, \sigma)=1-\left\{1-\exp \left[-\exp \left(-\frac{x-\mu}{\sigma}\right)\right]\right\}^{\alpha}
$$

for $-\infty<x<\infty, \mu \in \mathbb{R}$ and $\sigma>0$.

In this paper, we propose a new extended gamma generalized (EGG) model and obtain some of its structural properties. Given a continuous cdf $G(x)$ defined by (5), the EGG model is defined by

$$
F(x ; a, b, c, \beta, \lambda)=\left\{1-\left[1-\frac{\gamma\left(\beta,(\lambda x)^{c}\right)}{\Gamma(\beta)}\right]^{a}\right\}^{b},
$$

where $a, b, c, \beta>0$ are shape parameters and $\lambda>0$ is a scale parameter.

The pdf corresponding to (6) is given by

$$
\begin{aligned}
f(x ; a, b, c, \beta, \lambda) & =\frac{a b c \lambda^{c \beta}}{\Gamma(\beta)} x^{c \beta-1} \mathrm{e}^{-(\lambda x)^{c}}\left[1-\frac{\gamma\left(\beta,(\lambda x)^{c}\right)}{\Gamma(\beta)}\right]^{a-1} \\
& \times\left\{1-\left[1-\frac{\gamma\left(\beta,(\lambda x)^{c}\right)}{\Gamma(\beta)}\right]^{a}\right\}^{b-1} .
\end{aligned}
$$

The associated hrf is

$$
\begin{aligned}
\tau(x ; a, b, c, \beta, \lambda) & =\frac{a b c \lambda^{c \beta}}{\Gamma(\beta)} x^{c \beta-1} \mathrm{e}^{-(\lambda x)^{c}}\left[1-\frac{\gamma\left(\beta,(\lambda x)^{c}\right)}{\Gamma(\beta)}\right]^{a-1} \\
& \times\left\{1-\left[1-\frac{\gamma\left(\beta,(\lambda x)^{c}\right)}{\Gamma(\beta)}\right]^{a}\right\}^{b-1} \\
& \times\left\{1-\left[1-\left(1-\frac{\gamma\left(\beta,(\lambda x)^{c}\right)}{\Gamma(\beta)}\right)^{a}\right]^{b-1}\right\}^{-1} .
\end{aligned}
$$

Henceforth, $X$ stands for a random variable having the EGG density function (7).

Plots of the density and hrf of the EGG distribution for selected parameter values are displayed in Figures 1 and 2. 
(a)

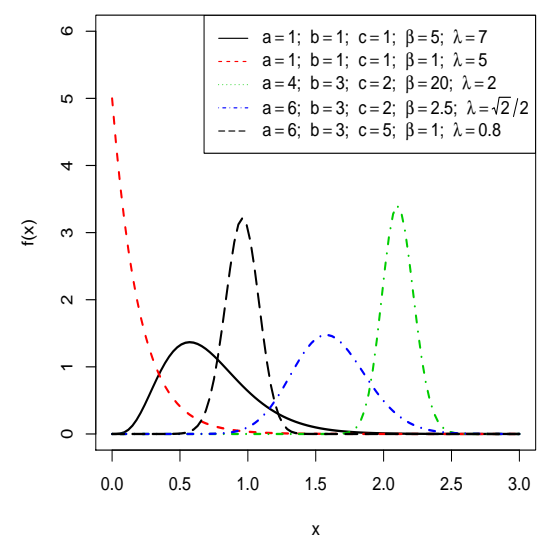

(b)

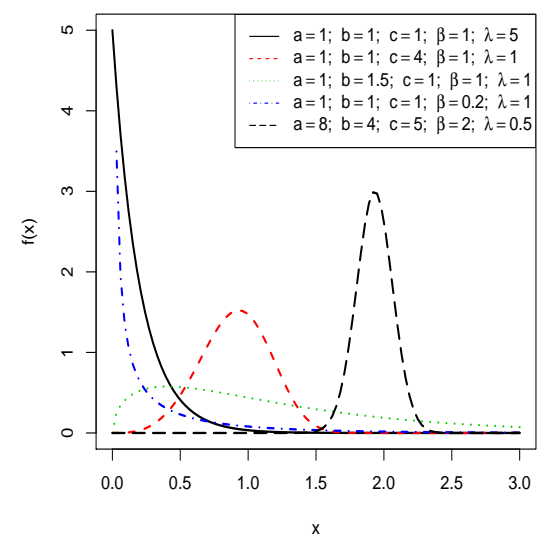

Figure 1: Plots of the EGG density function for some parameter values.

The EGG density function (7) allows for greater exibility of its tails and can be widely applied in many areas of engineering and biology. We study some structural properties of the pdf (7) because it extends several well-known distributions in the literature. We will study in the next sections some mathematical properties.

The rest of the paper is organized as follows. Section 2 provides some general useful expansions for the EGG density function. Moments and generating function of the EGG distribution are derived in Section 3. Quantile function and mean deviation are obtained in Section 4 and 5, respectively. The Rényi entropy and the reliability are determined in Sections 6 and 7, respectively. Order statistics are investigated in Section 8. Maximum likelihood estimation is addressed in Section 9. An application to a real data set is performed in Section 10. Some concluding remarks are addressed in Section 11.

\section{Useful Expansions}

Some useful expansions for (1) and (2) can be derived using the concept of exponentiated distributions. For an arbitrary baseline cdf $G(x)$, a random variable is said to have the exponentiated-G ("exp-G" for short) distribution 
(a)

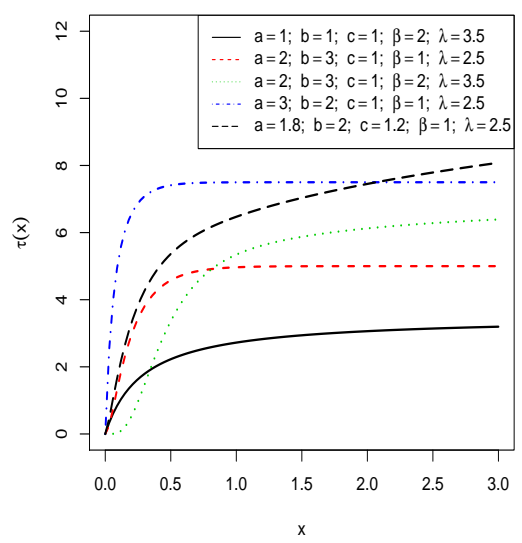

(c)

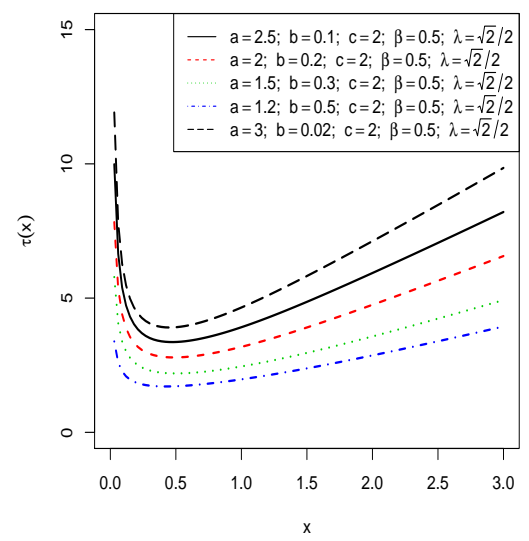

(b)

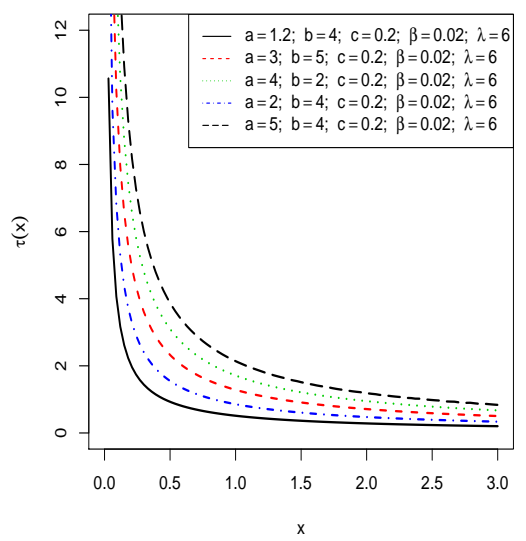

(d)

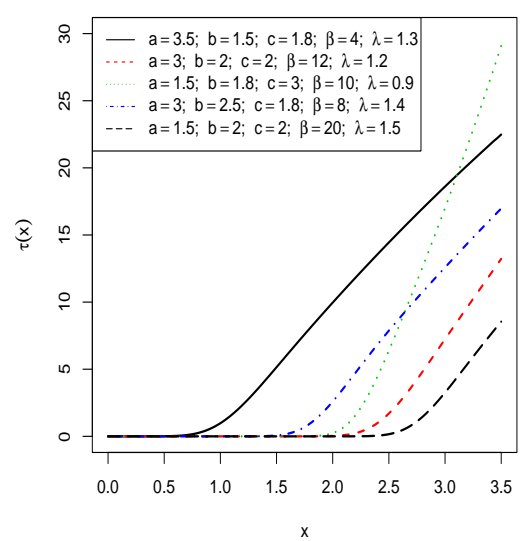

Figure 2: Plots of the hrf of the EGG distribution for some parameter values.

with power parameter $a>0$, say $T \sim \exp -\mathrm{G}(a)$, if the pdf and cdf of $T$ are

$$
h_{a}(x)=a g(x) G^{a-1}(x) \quad \text { and } \quad H_{a}(x)=G^{a}(x),
$$

respectively. The properties of the exponentiated distributions have been studied by many authors in recent years, see Mudholkar and Srivastava [9] and Mudholkar et al. [10] for exponentiated Weibull, Gupta et al. [6] and Gupta and Kundu [7] for exponentiated exponential, Nadarajah and Gupta [12] for 
Table 1: Some particular cases of the EGG distribution.

\begin{tabular}{|c|c|c|c|c|c|c|}
\hline \multicolumn{7}{|c|}{$a=b=1$} \\
\hline Case & $c$ & & $\bar{\lambda}$ & & $\beta$ & Distribution \\
\hline (1) & 1 & & $\bar{\lambda}$ & & $\beta$ & Gamma \\
\hline$(2)$ & 1 & & $\frac{1}{2}$ & & $\frac{n}{2}$ & Chi-square \\
\hline (3) & 1 & & $\lambda$ & & 1 & Exponential \\
\hline (4) & $c$ & & $\lambda$ & & 1 & Weibull \\
\hline (5) & 2 & & $\lambda$ & & 1 & Rayleigh \\
\hline (6) & 2 & & $\lambda$ & & $\frac{3}{2}$ & Maxwell \\
\hline (7) & 2 & & $\frac{1}{\sqrt{ }}$ & & $\frac{1}{2}$ & Folded normal \\
\hline (8) & $c$ & & $\lambda$ & & $\infty$ & Log-normal \\
\hline \multicolumn{7}{|c|}{$b=1$} \\
\hline & $\bar{a}$ & $c$ & & $\lambda$ & $\beta$ & \\
\hline$(9)$ & $\bar{a}$ & 1 & & $\lambda$ & $\beta$ & Exponentiated gamma \\
\hline (10) & $a$ & 1 & & $\frac{1}{2}$ & $\frac{n}{2}$ & Exponentiated chi-square \\
\hline (11) & $a$ & 1 & & 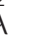 & 1 & Exponentiated exponential \\
\hline (12) & $a$ & $c$ & & $\lambda$ & 1 & Exponentiated Weibull \\
\hline (13) & $a$ & 2 & & $\lambda$ & 1 & Exponentiated Rayleigh \\
\hline (14) & $a$ & 2 & & $\lambda$ & $\frac{3}{2}$ & Exponentiated Maxwell \\
\hline$(15)$ & $a$ & 2 & & $\frac{1}{2}$ & $\frac{1}{2}$ & Exponentiated folded normal \\
\hline \multirow[t]{2}{*}{$(16)$} & $a$ & $c$ & & $\lambda$ & $\infty$ & Exponentiated log-normal \\
\hline & $a$ & $b$ & $c$ & $\bar{\lambda}$ & $\beta$ & \\
\hline$(17)$ & $a$ & $b$ & 1 & $\lambda$ & $\beta$ & Extended gamma \\
\hline (18) & $a$ & $b$ & 1 & $\frac{1}{2}$ & $\frac{n}{2}$ & Extended chi-square \\
\hline (19) & $a$ & $b$ & 1 & $\lambda$ & 1 & Extended exponential \\
\hline$(20)$ & $a$ & $b$ & $c$ & $\lambda$ & 1 & Extended Weibull \\
\hline$(21)$ & $a$ & $b$ & 2 & $\lambda$ & 1 & Exptended Rayleigh \\
\hline$(22)$ & $a$ & $b$ & 2 & $\lambda$ & $\frac{3}{2}$ & Extended Maxwell \\
\hline$(23)$ & $a$ & $b$ & 2 & $\frac{1}{\sqrt{2}}$ & $\frac{1}{2}$ & Extended folded normal \\
\hline$(24)$ & $a$ & $b$ & $c$ & $\lambda$ & $\infty$ & Extended log-normal \\
\hline
\end{tabular}

exponentiated gamma and Cordeiro et al. [2] for exponentiated generalized gamma distribution.

We consider the power series expansion

$$
(1-z)^{b}=\sum_{k=0}^{\infty}(-1)^{k}\left(\begin{array}{l}
b \\
k
\end{array}\right) z^{k},
$$

valid for any real non-integer $b$ and $|z|<1$. Using the generalized binomial (10) twice in equation (1), we can write the EGG cumulative distribution as

$$
F(x)=\sum_{k=0}^{\infty} v_{k+1} H_{k+1}(x),
$$


where $v_{k+1}=\sum_{j=1}^{\infty}(-1)^{k+j+1}\left(\begin{array}{c}b \\ j\end{array}\right)\left(\begin{array}{c}j a \\ k+1\end{array}\right)$ and $H_{k+1}(x)$ is the exp-G cdf with power parameter $k+1$. We can prove using Maple that $\sum_{k=0}^{\infty} v_{k+1}=1$, as expected.

By differentiating (11), we obtain

$$
f(x)=\sum_{k=0}^{\infty} v_{k+1} h_{k+1}(x),
$$

where $h_{k+1}(x)$ is the exp-G pdf with power parameter $k+1$. Equation (12) reveals that the EGG density function is a linear combination of exp-G densities. So, several mathematical properties of the EGG distribution such as ordinary and incomplete moments, moment generating function (mgf) and mean deviations can be obtained by knowing those of the exp-G distribution.

An integer power of the GG cumulative distribution can be expanded as

$$
G^{r}(x ; c, \beta, \lambda)=\frac{(\lambda x)^{r c \beta}}{\Gamma(\beta)^{r}} \sum_{m=0}^{\infty} c_{r, m}\left(\lambda x^{c}\right)^{m},
$$

where the quantities $c_{r, m}$ (for $m=1,2, \ldots$ ) are easily determined recursively from $c_{j, 0}=a_{0}^{j}$ and

$$
c_{j, i}=\left(i a_{0}\right)^{-1} \sum_{m=1}^{i}[(j+1) m-i] a_{m} c_{j, i-m},
$$

where $a_{m}=(-1)^{m} /[(a+m) m !]$. The coefficients $c_{j, i}$ for any $i$ can be calculated directly from $c_{j, 0}, \ldots, c_{j, i-1}$ and, therefore, from $a_{0}, \ldots, a_{i}$.

From equations (4), (12) and (13), we obtain an expansion for the EGG density function

$$
f(x ; a, b, c, \beta, \lambda)=\sum_{j, k, m=0}^{\infty} w_{j, k, m} g(x ; c,(k+1) \beta+m, \lambda),
$$

where

$$
w_{j, k, m}=w_{j, k, m}(a, b, c, \beta, \lambda)=\frac{(-1)^{j+k} a b \lambda^{m(1-c)}\left(\begin{array}{c}
b-1 \\
j
\end{array}\right)\left(\begin{array}{c}
(j+1) a-1 \\
k
\end{array}\right) c_{k, m}}{\Gamma(\beta)^{k}} .
$$

Equation (14) is the main result of this section. It allows us to derive some EGG mathematical properties from those properties of the GG distribution. 


\section{Moments and Generating Function}

Based on equation (14) we obtain the sth moment of $X$ as

$$
E\left(X^{s}\right)=\lambda^{-s} \sum_{j, k, m=0}^{\infty} w_{j, k, m} \frac{\Gamma[(k+1) \beta+m+s / c]}{\Gamma[(k+1) \beta+m]} .
$$

The moments of the EGG distribution can be expressed as linear functions of the corresponding GG moments. These expansions are readily computed numerically using standard statistical software. They (and other expansions in this paper) can also be evaluated in symbolic computation software such as Mathematica and Maple. In numerical applications, a large natural number $N$ can be used in the sums instead of infinity.

For lifetime models, it is usually of interest to compute the $k$ th incomplete moment of $X$ defined by $m_{k}(z)=\int_{0}^{z} x^{k} f(x) d x$. The quantity $m_{k}(z)$ can be calculated from (12) as

$$
m_{k}(z)=\sum_{j=0}^{\infty} p_{j} \int_{0}^{z} x^{k} g(x) G^{j}(x) d x,
$$

where $p_{j}=v_{j+1}(j+1)$.

Setting $u=G(x)$, we can write

$$
m_{k}(z)=\sum_{j=0}^{\infty} p_{j} \vartheta(z)
$$

where

$$
\vartheta(z)=\int_{0}^{G(z)} Q_{G}^{k}(u) u^{j} d u
$$

The quantity $\vartheta(z)$ can be computed numerically.

We provide explicit expressions for the $\operatorname{mgf} M(t)=E\left(\mathrm{e}^{\mathrm{tX}}\right)$ of $X$. First, the $\operatorname{mgf}$ of the $\operatorname{GG}(c, \xi, \lambda)$ distribution is given by Stacy and Mihram [17]

$$
M_{c, \xi, \lambda}(s)=\frac{1}{\Gamma(\xi)} \sum_{q=0}^{\infty} \Gamma\left(\frac{q}{c}+\xi\right) \frac{(s / \lambda)^{q}}{q !} .
$$

It follows from (14) and the GG generating function with $\xi=(k+1) \beta+m$

$$
M(t)=\sum_{j, k, m, q=0}^{\infty} w_{j, k, m} M_{c,(k+1) \beta+m, \lambda}(t) .
$$


Clearly, special formulas for the mgf of all sub-models of the EGG distribution can be easily derived from (18) by substitution of known parameters.

\section{Quantile Function}

The EGG quantile function, say $Q(u)=F^{-1}(u)$, is straightforward to be computed by inverting (6) provided a closed-form expression for the baseline quantile function $Q_{G G}(u)=G_{c, \beta, \lambda}^{-1}(u)$ is available. From equation (6), we can write

$$
Q(u)=Q_{G G}\left(1-\left[1-u^{1 / b}\right]^{1 / a}\right)
$$

A random variable $Y$ having density function (4) is denoted by $Y \sim \operatorname{GG}(c, \beta, \lambda)$. The GG quantile function $\left(Q_{G G}(u)\right)$ can be expressed in terms of the gamma quantile function $\left(Q_{G}(u)\right)$.

$$
Q_{G G}(u)=\lambda^{-1}\left[\lambda Q_{G}(u)\right]^{1 / c} .
$$

Plots of the EGG skewness and kurtosis as function of $a$ for selected values $b, c, \beta, \lambda$ and as function of $b$ for for selected values $a, c, \beta, \lambda$ are displayed in Figures 3 and 4 .
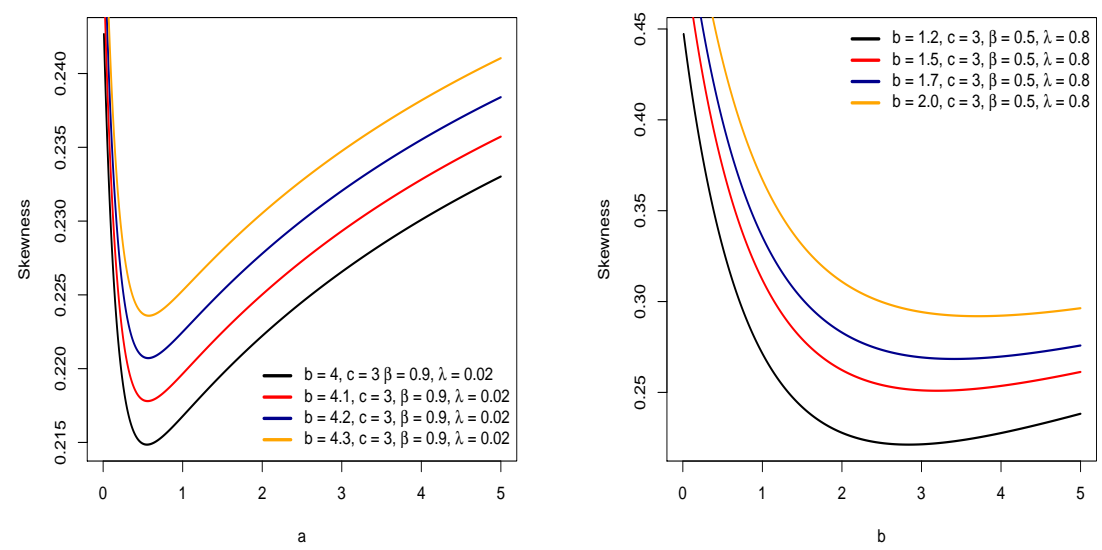

Figure 3: Plots of the EGG skewness for some parameter values. 

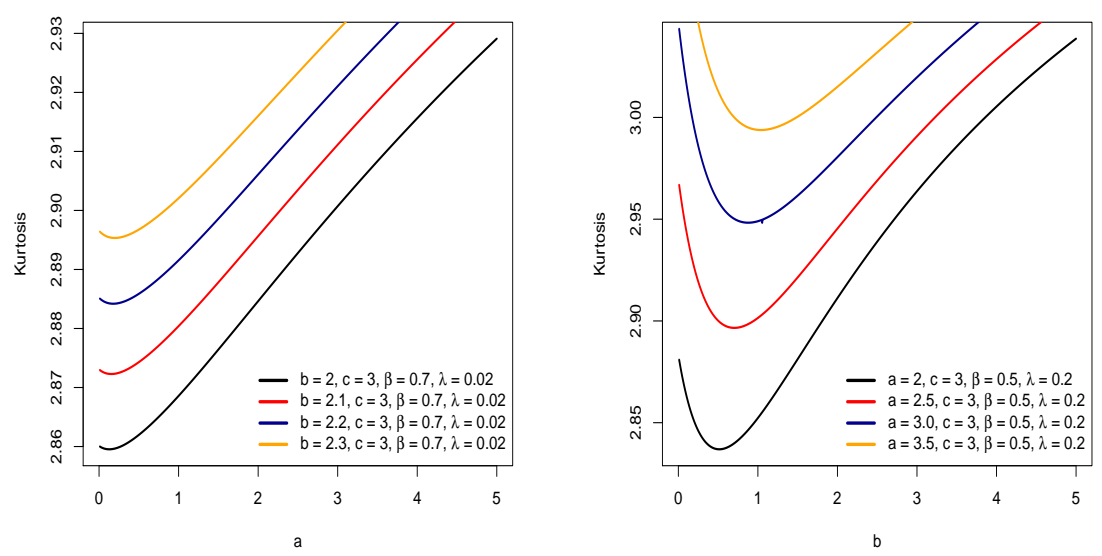

Figure 4: Plots of the EGG kurtosis for some parameter values.

Here, we derive a power series for the quantile function (19). If the baseline quantile function $Q_{G}(u)=G^{-1}(u)$ does not have a closed-form expression, it can usually be expressed in terms of a power series

$$
Q_{G}(u)=\sum_{i=0}^{\infty} a_{i} u^{i},
$$

where the coefficients $a_{i}$ are suitably chosen real numbers which depend on the parameters of the $\mathrm{G}$ distribution. For several important distributions, such as the normal, Student $t$, gamma, generalized gamma and beta distributions, $Q_{G}(u)$ does not have explicit expressions, but it can be expanded as in equation (20). As a simple example, for the normal $N(0,1)$ distribution, $a_{i}=0$ for $i=0,2,4, \ldots$ and $a_{1}=1, a_{3}=1 / 6, a_{5}=7 / 120$ and $a_{7}=127 / 7560, \ldots$

Using the binomial expansion, we obtain

$$
\left[1-\left(1-u^{1 / b}\right)^{1 / a}\right]^{i}=\sum_{j=0}^{i} \sum_{k=0}^{\infty}(-1)^{j+k}\left(\begin{array}{c}
i \\
j
\end{array}\right)\left(\begin{array}{c}
j / a \\
k
\end{array}\right) u^{k / b},
$$

and then using (20), the EGG quantile function can be expressed as

$$
Q(u)=\sum_{i=0}^{\infty} \sum_{j=0}^{i} \sum_{k=0}^{\infty}(-1)^{j+k} a_{i}\left(\begin{array}{c}
i \\
j
\end{array}\right)\left(\begin{array}{c}
j / a \\
k
\end{array}\right) u^{k / b}=\sum_{k=0}^{\infty} g_{k} u^{k / b},
$$


where

$$
g_{k}=\sum_{i=0}^{\infty} \sum_{j=0}^{i}(-1)^{j+k} a_{i}\left(\begin{array}{c}
i \\
j
\end{array}\right)\left(\begin{array}{c}
j / b \\
k
\end{array}\right) .
$$

For $0<u<1$, we have an expansion for $u^{\rho}$ which holds for $\rho>0$ real non-integer

$$
u^{\rho}=\sum_{l=0}^{\infty} s_{l}(\rho) u^{l}
$$

where

$$
s_{l}(\rho)=\sum_{m=l}^{\infty}(-1)^{m+l}\left(\begin{array}{c}
\rho \\
m
\end{array}\right)\left(\begin{array}{c}
m \\
l
\end{array}\right) .
$$

Setting $\rho=k / b$ in (22) and substituting in (21), we can write

$$
Q(u)=\sum_{k=0}^{\infty} g_{k} \sum_{l=0}^{\infty} s_{l}(k / b) u^{l}=\sum_{l=0}^{\infty} q_{l} u^{l},
$$

where $q_{l}=\sum_{k=0}^{\infty} g_{k} s_{l}(k / b)$.

Equation (23) is the main result of this section since it allows to obtain various mathematical quantities for the EGG distribution as proved in the next sections. The formula derived throughout the paper can be easily handled in most symbolic computation software platforms such as Maple and Mathematica since they have currently the ability to deal with analytic expressions of formidable size and complexity. Established explicit expressions to calculate statistical measures can be more efficient than computing them directly by numerical integration which can be prone to rounding of errors among others. The infinity limit in these sums can be substituted by a large positive integer such as 30 or 50 for most practical purposes.

\section{Mean Deviations}

The mean deviations about the mean $\delta_{1}(X)=E\left(\left|X-\mu_{1}^{\prime}\right|\right)$ and about the median $\delta_{2}(X)=E(|X-M|)$ of $X$ can be expressed as

$$
\delta_{1}(X)=2 \mu_{1}^{\prime} F\left(\mu_{1}^{\prime}\right)-2 m_{1}\left(\mu_{1}^{\prime}\right) \quad \text { and } \quad \delta_{2}(X)=\mu_{1}^{\prime}-2 m_{1}(M),
$$

respectively, where $F\left(\mu_{1}^{\prime}\right)$ is easily calculated from the cdf (6), $\mu_{1}^{\prime}=E(X)$ comes from (16) with $s=1, m_{1}(z)=\int_{0}^{z} x f(x) d x$ is the first 
incomplete moment of $X$ given by

$$
\begin{aligned}
m_{1}(z) & =\lambda^{-1} \sum_{j, k, m=0}^{\infty} w_{j, k, m}\left\{\frac{\Gamma[(k+1) \beta+m+1 / c]}{\Gamma[(k+1) \beta+m]}\right. \\
& \left.-\frac{\gamma\left[(k+1) \beta+m+1 / c, \lambda^{c} s^{c}\right]}{\Gamma[(k+1) \beta+m]}\right\}
\end{aligned}
$$

and $M$ is the median. Using (19), it follows that

$$
M=G^{-1}\left[\left(1-2^{\frac{-1}{b}}\right)^{\frac{1}{a}}\right],
$$

where $G^{-1}$ is the quantile function of (5).

Applications of (24) can be addressed to obtain Bonferroni and Lorenz curves defined for a given probability $\pi$ defined by $B(\pi)=m_{1}(1) /\left(\pi \mu_{1}^{\prime}\right)$ and $L(\pi)=m_{1}(q) / \mu_{1}^{\prime}$, respectively, where $q=Q(\pi)$ comes from (19).

\section{The Rényi Entropy}

The entropy of a random variable $X$ with density function $f(x)$ is a measure of variation of the uncertainty. One of the popular entropy measures is the Rényi entropy [15]. A large value of the entropy indicates the greater uncertainty in the data. The Rényi entropy is defined by (for $\gamma>0$ and $\gamma \neq 1$ )

$$
\mathcal{J}_{R}(\gamma)=\frac{1}{1-\gamma} \log \left(\int_{0}^{\infty} f^{\gamma}(x) d x\right) .
$$

Based on the pdf (7), the Rényi entropy of the EGG distribution is given by

$$
\begin{aligned}
\mathcal{J}_{R}(\gamma) & =\frac{1}{1-\gamma} \log \left(\int_{0}^{\infty} b^{\gamma} h_{a}^{\gamma}(x) H_{a}^{(b-1) \gamma}(x) d x\right) \\
& =\frac{1}{1-\gamma} \log \left(b^{\gamma} I_{k}\right),
\end{aligned}
$$

where

$$
I_{k}=\int_{0}^{\infty} h_{a}^{\gamma}(x) H_{a}^{(b-1) \gamma}(x) d x .
$$

We can calculate the Rényi entropy using the integral

$$
\begin{aligned}
\int_{0}^{\infty} f^{\gamma}(x) d x & =(a b)^{\gamma} \int_{0}^{\infty} g^{\gamma}(x)[1-G(x)]^{(a-1) \gamma} \\
& \times\left\{1-[1-G(x)]^{a}\right\}^{(b-1) \gamma} d x
\end{aligned}
$$


and then expanding the binomial we can write

$$
\begin{aligned}
\int_{0}^{\infty} f^{\gamma}(x) d x & =(a b)^{\gamma} \int_{0}^{\infty} g^{\gamma}(x) \sum_{j, k=0}^{\infty}(-1)^{j+k}\left(\begin{array}{c}
(b-1) \gamma \\
j
\end{array}\right) \\
& \times\left(\begin{array}{c}
(j+\gamma) a-\gamma \\
k
\end{array}\right) G^{k}(x) d x .
\end{aligned}
$$

Substituting (4) and (13) in equation (28), we obtain

$$
\begin{aligned}
\int_{0}^{\infty} f^{\gamma}(x) d x & =\left[\frac{a b c}{\Gamma(\beta)}\right]^{\gamma} \sum_{j, k, m=0}^{\infty} \frac{(-1)^{j+k}\left(\begin{array}{c}
\left(\begin{array}{c}
b-1) \gamma \\
j
\end{array}\right)\left(\begin{array}{c}
(j+\gamma) a-\gamma \\
k
\end{array}\right) \\
\Gamma(\beta)^{k}
\end{array}\right.}{} \times \lambda^{c[(k+\gamma) \beta+m]+m(1-c)} \mathcal{I}
\end{aligned}
$$

where

$$
\begin{aligned}
\mathcal{I} & =\int_{0}^{\infty} x^{c[(k+\gamma) \beta+m]} \mathrm{e}^{-\gamma(\lambda x)^{c}} d x \\
& =\gamma^{-[(k+\gamma) \beta+m+1 / c]} \lambda^{-\{c[(k+\gamma) \beta+m]+1\}} \Gamma[(k+\gamma) \beta+m+1 / c]
\end{aligned}
$$

Thus,

$$
\begin{aligned}
\int_{0}^{\infty} f^{\gamma}(x) d x & =\frac{a^{\gamma} b^{\gamma} c^{\gamma-1}}{\Gamma(\beta)^{\gamma}} \sum_{j, k, m=0}^{\infty}(-1)^{j+k}\left(\begin{array}{c}
(b-1) \gamma \\
j
\end{array}\right) \\
& \times\left(\begin{array}{c}
(j+\gamma) a-\gamma \\
k
\end{array}\right) \lambda^{m(1-c)-1} \gamma^{-[(k+\gamma) \beta+m+1 / c]} \\
& \times \frac{\Gamma[(k+\gamma) \beta+m+1 / c]}{\Gamma(\beta)^{k}}
\end{aligned}
$$

Equation (29) is the main result of this section.

\section{Reliability}

The component fails at the instant that the random stress $X_{2}$ applied to it exceeds the random strength $X_{1}$, and the component will function satisfactorily whenever $X_{1}>X_{2}$. Hence, $R=P\left(X_{2}<X_{1}\right)$ is a measure of component reliability. It has many applications especially in the area of engineering. We derive the reliability $R$ when $X_{1}$ and $X_{2}$ have independent $\operatorname{EGG}\left(a_{1}, b_{1}, c_{1}, \beta_{1}, \lambda_{1}\right)$ and $\operatorname{EGG}\left(a_{2}, b_{2}, c_{2}, \beta_{2}, \lambda_{2}\right)$ distributions with the same parameter vector $\eta$ for $G$. The reliability is defined by 


$$
R=\int_{0}^{\infty} f_{1}(x) F_{2}(x) d x .
$$

The pdf of $X_{1}$ and cdf of $X_{2}$ are obtained from equations (14) and (11) as

$$
\begin{aligned}
& f_{1}(x)=g(x ; \eta) \sum_{k=0}^{\infty} p_{k}\left(a_{1}, b_{1}, c_{1}\right) G(x ; \eta)^{(k+1) a_{1}-1} \\
& \text { and } \quad F_{2}(x)=\sum_{q=0}^{\infty} w_{q}\left(b_{2}, c_{2}\right) G(x ; \eta)^{(q+1) a_{2}},
\end{aligned}
$$

where

$$
p_{k}\left(a_{1}, b_{1}, c_{1}\right)=(k+1) a_{1} w_{k}\left(b_{1}, c_{1}\right)
$$

and

$$
w_{q}\left(b_{2}, c_{2}\right)=\frac{(-1)^{k} b_{2} c_{2}}{q+1} \sum_{j=0}^{\infty}(-1)^{j}\left(\begin{array}{c}
c_{2}-1 \\
j
\end{array}\right)\left(\begin{array}{c}
(j+1) b_{2}-1 \\
q
\end{array}\right) .
$$

Hence,

$$
\begin{aligned}
R & =\sum_{k=0}^{\infty} p_{k}\left(a_{1}, b_{1}, c_{1}\right) w_{q}\left(b_{2}, c_{2}\right) \\
& \left.\times \int_{-\infty}^{\infty} g(x ; \eta) G(x ; \eta)\right)^{(k+1) a_{1}+(q+1) a_{2}-1} d x .
\end{aligned}
$$

Setting $u=G(x)$, we have

$$
R=\sum_{k=0}^{\infty} p_{k}\left(a_{1}, b_{1}, c_{1}\right) w_{q}\left(b_{2}, c_{2}\right) \int_{0}^{1} u^{(k+1) a_{1}+(q+1) a_{2}-1} d u .
$$

Hence, the reliability of the EGG distribution reduces to

$$
R=\sum_{k=0}^{\infty} \frac{p_{k}\left(a_{1}, b_{1}, c_{1}\right) w_{q}\left(b_{2}, c_{2}\right)}{(k+1) a_{1}+(q+1) a_{2}} .
$$

\section{Order Statistics}

Order statistics make their appearance in many areas of statistical theory and practice. The density $f_{i: n}(x)$ of the $i$ th order statistic, for $i=1, \ldots, n$, from 
independent and identically distributed random variables $X_{1}, \ldots, X_{n}$ from the EGG distribution is given by

$$
f_{i: n}(x)=\frac{f(x)}{B(i, n-i+1)} \sum_{r=0}^{n-i}(-1)^{r}\left(\begin{array}{c}
n-i \\
r
\end{array}\right) F(x)^{r+i-1} .
$$

We can write from equations (6) and (7)

$$
\begin{aligned}
f_{i: n}(x)= & \frac{1}{B(i, n-i+1)} \sum_{r=0}^{n-i}(-1)^{r}\left(\begin{array}{c}
n-i \\
r
\end{array}\right) \sum_{k=0}^{\infty}(k+1) a w_{k} g(x) \\
& \times \sum_{l, m=0}^{\infty}(-1)^{l+m}\left(\begin{array}{c}
l b \\
m
\end{array}\right)\left(\begin{array}{c}
(r+i-1) c \\
l
\end{array}\right) G^{(k+m+1) a-1} \\
= & \frac{1}{B(i, n-i+1)} \sum_{r=0}^{n-i} \sum_{k, l, m=0}^{\infty}(-1)^{r+l+m}\left(\begin{array}{c}
n-i \\
r
\end{array}\right)\left(\begin{array}{c}
l b \\
m
\end{array}\right) \\
& \times\left(\begin{array}{c}
(r+i-1) c \\
l
\end{array}\right) p_{k} g(x) G^{(k+m+1) a-1} \\
= & \sum_{k, l, m=0}^{\infty} q_{k, l, m} g(x) G^{(k+m+1) a-1},
\end{aligned}
$$

where

$$
q_{k, l, m}=\sum_{r=0}^{n-i} \frac{(-1)^{r+l+m}\left(\begin{array}{c}
n-i \\
r
\end{array}\right)\left(\begin{array}{c}
l b \\
m
\end{array}\right)\left(\begin{array}{c}
(r+i-1) c \\
l
\end{array}\right) p_{k}}{B(i, n-i+1)} .
$$

Another way to write order statistic is given by

$$
\begin{aligned}
f_{i: n}(x)= & \frac{a b g(x)}{B(i, n-i+1)} \sum_{r=0}^{n-i} \sum_{k, l=0}^{\infty}(-1)^{r+k+l}\left(\begin{array}{c}
n-i \\
r
\end{array}\right) \\
& \times\left(\begin{array}{c}
b(r+i)-1 \\
k
\end{array}\right)\left(\begin{array}{c}
a(k+1)-1 \\
l
\end{array}\right) G^{l} .
\end{aligned}
$$

Using the equation (13) we can write (32) as

$$
f_{i: n}(x)=\sum_{k, l, m=0}^{\infty} s_{k, l, m} g\left[x ; c, m+\beta\left(r l c^{-1}+1\right), \lambda\right]
$$


where

$$
\begin{aligned}
s_{k, l, m}= & \sum_{r=0}^{n-i} \frac{(-1)^{r+k+l} a b\left(\begin{array}{c}
n-i \\
r
\end{array}\right)\left(\begin{array}{c}
b(r+i)-1 \\
k
\end{array}\right)\left(\begin{array}{c}
a(k+1)-1 \\
l
\end{array}\right) \lambda^{m(1-c)} c_{l, m}}{B(i, n-i+1) \Gamma(\beta)^{l+1}} \\
& \times \Gamma\left[m+\beta\left(r l c^{-1}+1\right)\right] .
\end{aligned}
$$

Equations (31) and (32) are the main results of this section.

\section{Estimation}

We determine the maximum likelihood estimates (MLEs) of the parameters of the EGG distribution from complete samples only. Let $x_{1}, \ldots, x_{n}$ be a random sample of size $n$ from the $\operatorname{EGG}(a, b, c, \beta, \lambda)$ distribution. The log-likelihood function for the vector of parameters $\theta=(a, b, c, \beta, \lambda)^{T}$ can be expressed as

$$
\begin{aligned}
l(\theta) & =n \log \left[\frac{a b c \lambda^{c \beta}}{\Gamma(\beta)}\right]+(c \beta-1) \sum_{i=1}^{n} \log \left(x_{i}\right) \\
& -\lambda^{c} \sum_{i=1}^{n} x_{i}^{c}+(a-1) \sum_{i=1}^{n} \log \left[1-\frac{\gamma\left(\beta,(\lambda x)^{c}\right)}{\Gamma(\beta)}\right] \\
& +(b-1) \sum_{i=1}^{n} \log \left\{1-\left[1-\frac{\gamma\left(\beta,(\lambda x)^{c}\right)}{\Gamma(\beta)}\right]^{a}\right\} .
\end{aligned}
$$

The components of the score vector $U(\theta)$ are given by

$$
\begin{aligned}
& U_{a}(\theta)= \frac{n}{a}+\sum_{i=1}^{n} \log \left[\varphi\left(x_{i}\right)\right]-(b-1) \sum_{i=1}^{n} \frac{\varphi^{a}\left(x_{i}\right) \log \left[\varphi\left(x_{i}\right)\right]}{1-\varphi^{a}\left(x_{i}\right)}, \\
& U_{b}(\theta)=\frac{n}{b}+\sum_{i=1}^{n} \log \left\{1-\varphi^{a}\left(x_{i}\right)\right\} \\
& U_{c}(\theta)=\frac{n}{c}+\beta \log (\lambda)+\beta \sum_{i=1}^{n} \log \left(x_{i}\right)-\lambda^{c} \sum_{i=1}^{n} x_{i}^{c} \log \left(\lambda x_{i}\right) \\
&-\frac{\lambda^{c \beta}(a-1)}{\Gamma(\beta)} \sum_{i=1}^{n} \frac{x_{i}^{c \beta} \mathrm{e}^{-\left(\lambda x_{i}\right)^{c}} \log \left(\lambda x_{i}\right)}{\varphi\left(x_{i}\right)}
\end{aligned}
$$




$$
\begin{aligned}
- & \frac{a \lambda^{c \beta}(b-1)}{\Gamma(\beta)} \sum_{i=1}^{n} \frac{x_{i}^{c \beta} \mathrm{e}^{-\left(\lambda x_{i}\right)^{c}} \varphi^{a}\left(x_{i}\right) \log \left(\lambda x_{i}\right)}{\varphi\left(x_{i}\right)\left\{-1+\varphi^{a}\left(x_{i}\right)\right\}}, \\
U_{\beta}(\theta) & =n[c \log (\lambda)-\psi(\beta)]+c \sum_{i=1}^{n} \log \left(x_{i}\right) \\
& -(a-1)\left\{n \psi(\beta)-\log \left(\lambda x_{i}\right)^{c}-\frac{1}{\Gamma(\beta)} \sum_{i=1}^{n} \frac{\eta\left(x_{i}\right)}{\varphi\left(x_{i}\right)}\right\} \\
& -a(b-1) \sum_{i=1}^{n} \frac{\varphi^{a-1}\left(x_{i}\right)}{1-\varphi^{a}\left(x_{i}\right)}\left\{\varphi\left(x_{i}\right)\left\{\psi(\beta)-\log \left(\lambda x_{i}\right)^{c}\right\}\right. \\
& \left.-\Gamma^{-1}(\beta) \eta\left(x_{i}\right)\right\},
\end{aligned}
$$

where

$$
\begin{aligned}
& \eta\left(x_{i}\right)=G_{2,3}^{3,0}\left(\begin{array}{l|l}
\left(\lambda x_{i}\right)^{c} & \begin{array}{l}
1,1 \\
0,0, \beta
\end{array}
\end{array}\right) \\
& U_{\lambda}(\theta)=\frac{n c \beta}{\lambda}-c \lambda^{c-1} \sum_{i=1}^{n} x_{i}^{c}-\frac{c(a-1) \lambda^{c \beta-1}}{\Gamma(\beta)} \sum_{i=1}^{n} \frac{x_{i}^{c \beta} \mathrm{e}^{-\left(\lambda x_{i}\right)^{c}}}{\varphi\left(x_{i}\right)} \\
& +\frac{a c(b-1) \lambda^{c \beta-1}}{\Gamma(\beta)} \sum_{i=1}^{n} \frac{x_{i}^{c \beta} \mathrm{e}^{-\left(\lambda x_{i}\right)^{c}} \varphi^{a-1}\left(x_{i}\right)}{1-\varphi^{a}\left(x_{i}\right)}
\end{aligned}
$$

where $\varphi\left(x_{i}\right)=\frac{\Gamma\left(\beta,\left(\lambda x_{i}\right)^{c}\right)}{\Gamma(\beta)}, \psi(\cdot)$ is the digamma function, $\Gamma(\cdot, \cdot)$ is the upper incomplete gamma function and the Meijer G-function defined by

$$
G_{p, q}^{m, n}\left(x \mid \begin{array}{l}
a_{1}, \ldots, a_{p} \\
b_{1}, \ldots, b_{q}
\end{array}\right)=\int \frac{\prod_{j=1}^{m} \Gamma\left(b_{j}+t\right) \prod_{j=1}^{n} \Gamma\left(1-a_{j}-t\right)}{\prod_{j=n+1}^{p} \Gamma\left(a_{j}+t\right) \prod_{j=m+1}^{p} \Gamma\left(1-b_{j}-t\right)} \frac{x^{-t}}{2 \pi i} d t
$$

where $\mathrm{i}=\sqrt{-1}$ is the complex unit and $\mathrm{L}$ denotes an integration path; see Section 9.3 in [5] for a description of this path. The Meijer G-function contains as particular cases many integrals with elementary and special functions [13]. For interval estimation on the model parameters, we require the observed information matrix $J(\theta)$, whose elements are given in Appendix A. Let $\widehat{\theta}$ be 
the MLE of $\theta$. Under standard regular conditions [4] which are fulfilled for the proposed model whenever the parameters are in the interior of the parameter space, we can approximate the distribution of $\sqrt{n}(\widehat{\theta}-\theta)$ by the multivariate normal $N_{5}\left(0, K(\theta)^{-1}\right)$, where $K(\theta)=\lim _{n \rightarrow \infty} n^{-1} J_{n}(\theta)$ is the unit information matrix. Based on the approximate multivariate normal $N_{5}\left(0, J(\widehat{\theta})^{-1}\right)$ distribution of $\widehat{\theta}$, where $J(\widehat{\theta})$ is the observed information matrix evaluated at $\widehat{\theta}$, we can construct approximate confidence regions for the model parameters.

\section{Application}

Here, we present an application of the EGG distribution to a real data set for illustrative purposes. This application indicates the flexibility of the new distribution in modeling positive data. All the computations were done using the $\mathrm{R}$ software [14]. These data on failure times are reported in the book "Weibull Models" by Murthy et al. [8], page 297:

$0.040,1.866,2.385,3.443,0.301,1.876,2.481,3.467,0.309,1.899,2.610,3.478$, $0.557,1.911,2.625,3.578,0.943,1.912,2.632,3.595,1.070,1.914,2.646,3.699$, $1.124,1.981,2.661,3.779,1.248,2.010,2.688,3.924,1.281,2.038,2.82,3,4.035$, $1.281,2.085,2.890,4.121,1.303,2.089,2.902,4.167,1.432,2.097,2.934,4.240$, $1.480,2.135,2.962,4.255,1.505,2.154,2.964,4.278,1.506,2.190,3.000,4.305$, $1.568,2.194,3.103,4.376,1.615,2.223,3.114,4.449,1.619,2.224,3.117,4.485$, $1.652,2.229,3.166,4.570,1.652,2.300,3.344,4.602,1.757,2.324,3.376,4.663$.

Table 2 lists the MLEs (and the corresponding standard errors in parentheses) of the unknown parameters of the EGG, beta generalized gamma (BGG), exponentiated gamma (EG), generalized gamma (GG) and gamma $(\mathrm{G})$ distributions.

Table 2: MLEs (standard errors in parentheses).

\begin{tabular}{llllll}
\hline Distributions & \multicolumn{5}{c}{ Estimates } \\
\hline EGG $(a, b, c, \beta, \lambda)$ & 0.1369697 & 2.4039437 & 2.4520172 & 0.0823543 & 0.0808677 \\
& $(0.020472)$ & $(0.639392)$ & $(0.048096)$ & $(0.047561)$ & $(0.024665)$ \\
BGG $(a, b, c, \beta, \lambda)$ & 0.7892168 & 1.6334571 & 30.708165 & 0.0691936 & 0.2045010 \\
& $(0.865362)$ & $(0.607154)$ & $(26.05629)$ & $(0.094823)$ & $(0.012077)$ \\
EG $(b, c, \beta, \lambda)$ & 0.0209191 & 4.2856360 & 18.734850 & 0.41951783 & \\
& $(0.025713)$ & $(2,520963)$ & $(25.935107)$ & $(0.2517966)$ & \\
GG $(c, \beta, \lambda)$ & 5.9604550 & 0.2776150 & 0.2393780 & & \\
& $(3.756108)$ & $(0.218047)$ & $(0.027935)$ & & \\
G $(\beta, \lambda)$ & 3.5283100 & 1.3768370 & & & \\
& $(0.517671)$ & $(0.217090)$ & & & \\
\hline
\end{tabular}


Next, we shall apply formal goodness-of-fit tests in order to verify which distribution fits better to these data. We consider the Cramér-von Mises $\left(W^{*}\right)$ and Anderson-Darling $\left(A^{*}\right)$ statistics described in Chen and Balakrishnan [1]. In general, the smaller the values of these statistics, the better the fit to the data. Let $L(x ; \theta)$ be the cdf, where the form of $L$ is known but $\theta$ (a $k$-dimensional parameter vector, say) is unknown. To obtain the statistics $W^{*}$ and $A^{*}$, we can proceed as follows: (i) Compute $v_{i}=L\left(x_{i} ; \widehat{\theta}\right)$, where the $x_{i}$ 's are in ascending order; (ii) Compute $y_{i}=\Phi^{-1}\left(v_{i}\right)$, where $\Phi(\cdot)$ is the standard normal cdf and $\Phi^{-1}(\cdot)$ its inverse; (iii) Compute $u_{i}=\Phi\left\{\left(y_{i}-\bar{y}\right) / s_{y}\right\}$, where $\bar{y}=(1 / n) \sum_{i=1}^{n} y_{i}$ and $s_{y}^{2}=(n-1)^{-1} \sum_{i=1}^{n}\left(y_{i}-\bar{y}\right)^{2}$; (iv) Calculate

$$
W^{2}=\sum_{i=1}^{n}\left\{u_{i}-\frac{(2 i-1)}{2 n}\right\}^{2}+\frac{1}{12 n}
$$

and

$$
A^{2}=-n-\frac{1}{n} \sum_{i=1}^{n}\left\{(2 i-1) \log \left(u_{i}\right)+(2 n+1-2 i) \log \left(1-u_{i}\right)\right\}
$$

(v) Modify $W^{2}$ into $W^{*}=W^{2}(1+0.5 / n)$ and $A^{2}$ into $A^{*}=A^{2}(1+0.75 / n+$ $\left.2.25 / n^{2}\right)$.

The statistics $A^{*}$ and $W^{*}$ for the fitted models are listed in Table 3 for the eruptions time data. First, the proposed EGG model fits these data better than the other models according to the statistics $A^{*}$ and $W^{*}$. The EGG model may be an interesting alternative to other models available in the literature for modeling positive real data. We compute the AIC, BIC and CAIC information criteria for the fitted models are listed in Table 4 for the eruptions time data.

Table 3: Statistics $A^{*}$ and $W^{*}$

\begin{tabular}{lcc}
\hline Distributions & $\mathbf{A}^{*}$ & $\mathbf{W}^{*}$ \\
\hline EGG & $\mathbf{0 . 5 4 0 7 5 8}$ & $\mathbf{0 . 0 7 8 2 3 4}$ \\
BGG & 0.713544 & 0.117482 \\
EG & 1.435337 & 0.164614 \\
GG & 0.766984 & 0.114399 \\
G & 1.367580 & 0.161986 \\
\hline
\end{tabular}


Table 4: Statistics AIC, CAIC and BIC

\begin{tabular}{lccc}
\hline Distributions & AIC & BIC & CAIC \\
\hline EGG & $\mathbf{2 6 5 . 6 9 5 6}$ & $\mathbf{2 6 6 . 4 5 5 1}$ & $\mathbf{2 7 7 . 9 0 8 8}$ \\
BGG & 259.6554 & 260.4149 & 271.8686 \\
EG & 264.0226 & 264.5226 & 273.7932 \\
GG & 263.8324 & 264.1287 & 271.1603 \\
G & 280.7907 & 280.9370 & 285.7660 \\
\hline
\end{tabular}

More information is provided by a visual comparison of the histogram of the data and the fitted densities. The plots of the fitted EGG, BGG, EG, GG, and $\mathrm{G}$ density functions are displayed in Figure 5. These plots indicate that the new distribution provides a better fit to these data.

\section{Conclusions}

We propose a new extended generalized gamma (EGG) distribution with two additional positive parameters to extend the generalized gamma (GG) distribution. The current generalization provides means of its continuous extensions to still more complex situations. The new distribution unifies more than 24 distributions and yields a general overview of these distributions for theoretical studies. We demonstrate that the probability density function (pdf) of the EGG distribution can be expressed as a linear combination of GG density functions. This result allows us to derive explicit expressions for several measures of the EGG distribution such as the ordinary moments, generating function and mean deviations. Explicit expressions for the Rényi entropy and reliability are given. We discuss maximum likelihood estimation and obtain the observed information matrix. The inference on the model parameters is based on Cramér-von Mises and Anderson-Darling statistics. An application of the EGG model to real data demonstrates its potentiality. We hope this generalization may attract several applications in statistics, biology, engineering and other areas.

\section{Appendix A - Observed Information Matrix}

The elements of the observed information matrix $J(\theta)$ for the parameters 


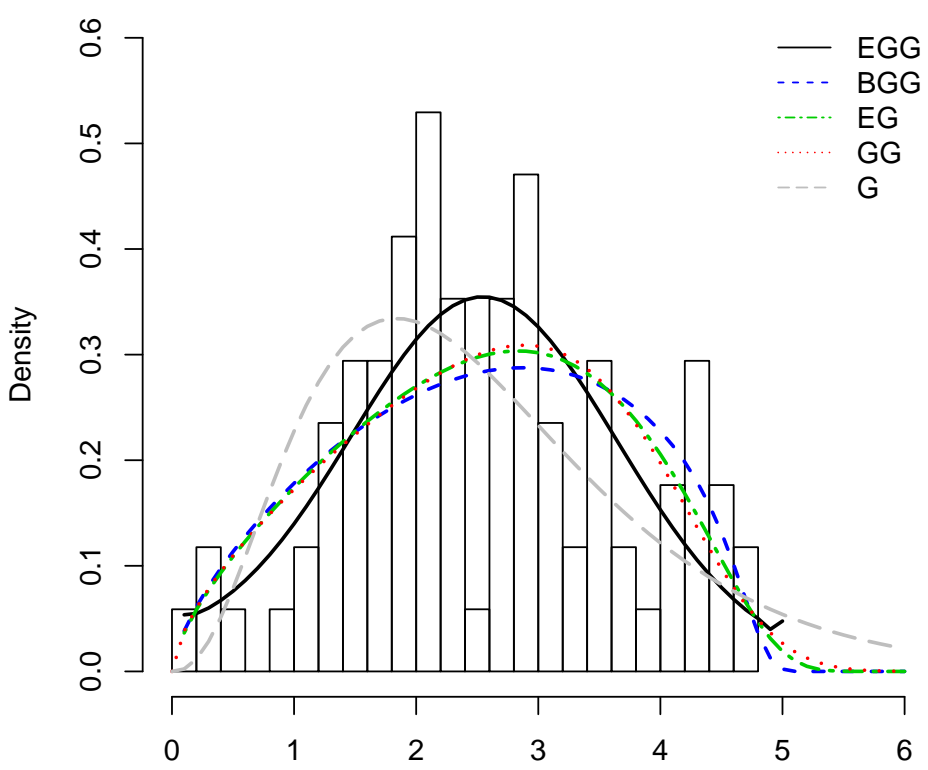

Figure 5: Fitted densities to the current data.

$(a, b, c, \eta)$ are:

$$
\begin{gathered}
U_{a, a}(\theta)=-\frac{n}{a^{2}}-(b-1) \sum_{i=1}^{n} \frac{\varphi^{a}\left(x_{i}\right) \log ^{2}\left[\varphi\left(x_{i}\right)\right]}{\left\{1-\varphi^{a}\left(x_{i}\right)\right\}^{2}}, \\
U_{a, b}(\theta)=-\sum_{i=1}^{n} \frac{\varphi^{a}\left(x_{i}\right) \log \left[\varphi\left(x_{i}\right)\right]}{1-\varphi^{a}\left(x_{i}\right)}, \\
U_{a, c}(\boldsymbol{\theta})=-\frac{\lambda^{c \beta}}{\Gamma(\beta)} \sum_{i=1}^{n} \frac{x_{i}^{c \beta} \mathrm{e}^{-\left(\lambda x_{i}\right)^{c}} \log \left(\lambda x_{i}\right)}{\varphi\left(x_{i}\right)} \\
+\frac{\lambda^{c \beta}(b-1)}{\Gamma(\beta)} \sum_{i=1}^{n}\left\{\frac{x_{i}^{c \beta} \mathrm{e}^{-\left(\lambda x_{i}\right)^{c}} \varphi^{a-1}\left(x_{i}\right) \log \left(\lambda x_{i}\right)}{\left\{1-\varphi^{a}\left(x_{i}\right)\right\}^{2}}\right.
\end{gathered}
$$




$$
\begin{aligned}
& \times\left\{1-\varphi^{a}\left(x_{i}\right)+a \log \left[\varphi\left(x_{i}\right)\right]\right\}, \\
U_{a, \beta}(\theta)= & -n \psi(\beta)+\log \left(\lambda x_{i}\right)^{c}+\frac{1}{\Gamma(\beta)} \sum_{i=1}^{n} \frac{\eta\left(x_{i}\right)}{\varphi\left(x_{i}\right)} \\
& -(b-1) \sum_{i=1}^{n} \frac{\gamma^{a-1}\left(\beta,(\lambda x)^{c}\right)}{1-\gamma^{a}\left(\beta,(\lambda x)^{c}\right)}\left\{a \rho\left(x_{i}\right) \log \left[\gamma\left(\beta,(\lambda x)^{c}\right)\right]\right. \\
+ & \left(\rho\left(x_{i}\right)+\left[1-\gamma\left(\beta,(\lambda x)^{c}\right)\right] \psi(\beta)\right) \\
\times & \left.\left(1+\frac{a \gamma^{a}\left(\beta,(\lambda x)^{c}\right) \log \left[\gamma\left(\beta,(\lambda x)^{c}\right)\right]}{1-\gamma^{a}\left(\beta,(\lambda x)^{c}\right)}\right)\right\},
\end{aligned}
$$

where

$$
\begin{aligned}
& \rho\left(x_{i}\right)=-\psi(\beta)+\varphi\left(x_{i}\right) \log \left(\lambda x_{i}\right)^{c}+\Gamma^{-1}(\beta) \eta\left(x_{i}\right)+\psi(\beta)\left[1-\varphi\left(x_{i}\right)\right], \\
& U_{a, \lambda}(\theta)=-\frac{\lambda^{c \beta-1}}{\Gamma(\beta)} \sum_{i=1}^{n} \frac{x_{i}^{c} \mathrm{e}^{-\left(\lambda x_{i}\right)^{c}}}{\varphi\left(x_{i}\right)}+\frac{c \lambda^{-1}(b-1)}{\Gamma(\beta)} \\
& \times \sum_{i=1}^{n} \frac{\varphi^{a-1}\left(x_{i}\right)\left\{a \lambda^{c \beta} x_{i}^{c \beta-2} \mathrm{e}^{-\left(\lambda x_{i}\right)^{c}} \log \varphi\left(x_{i}\right)+1+\varphi^{a}\left(x_{i}\right)\right\}}{\left[1-\varphi^{a}\left(x_{i}\right)\right]^{2}}, \\
& U_{b, b}(\theta)=-\frac{n}{b^{2}} \\
& U_{b, c}(\theta)=\frac{a \lambda^{c \beta}}{\Gamma(\beta)} \sum_{i=1}^{n} \frac{\varphi^{a-1}\left(x_{i}\right) \mathrm{e}^{-\left(\lambda x_{i}\right)^{c}} \log \left(\lambda x_{i}\right)}{1-\varphi^{a}\left(\lambda x_{i}\right)}, \\
& U_{b, \beta}(\theta)=a \sum_{i=1}^{n} \frac{\varphi^{a-1}\left(x_{i}\right)}{1-\varphi^{a}\left(x_{i}\right)}\left\{-\rho\left(x_{i}\right)-\psi(\beta)\left[1-\varphi\left(x_{i}\right)\right]\right\}, \\
& U_{b, \lambda}(\theta)=\frac{a c \lambda^{c \beta-1}}{\Gamma(\beta)} \sum_{i=1}^{n} \frac{x_{i}^{c \beta} \varphi^{a-1}\left(x_{i}\right) \mathrm{e}^{-\left(\lambda x_{i}\right)^{c}}}{1-\varphi^{a}\left(x_{i}\right)}
\end{aligned}
$$




$$
\begin{aligned}
U_{c, c}(\theta) & =-\frac{n}{c^{2}}-\lambda^{c} \log ^{2}(\lambda) \sum_{i=1}^{n} x_{i}^{c}-2 \lambda^{c} \log (\lambda) \sum_{i=1}^{n} x_{i}^{c} \log \left(x_{i}\right) \\
& -\lambda^{c} \sum_{i=1}^{n} x_{i}^{c} \log ^{2}\left(x_{i}\right)-\frac{\lambda^{c \beta}(a-1)}{\Gamma^{2}(\beta)} \sum_{i=1}^{n} x_{i}^{c \beta} \varphi^{-2}\left(x_{i}\right) \mathrm{e}^{-\left(\lambda x_{i}\right)^{c}} \\
& \times \log ^{2}\left(\lambda x_{i}\right)\left[\beta \Gamma(\beta) \varphi\left(x_{i}\right)-\left(\lambda x_{i}\right)^{c} \Gamma(\beta) \varphi\left(x_{i}\right)+\mathrm{e}^{-\left(\lambda x_{i}\right)^{c}}\right. \\
& \left.\times\left(\lambda x_{i}\right)^{c \beta}\right]+\frac{a \lambda^{c \beta}(b-1)}{\Gamma(\beta)} \sum_{i=1}^{n} \frac{x_{i}^{c \beta} \varphi^{a-1}\left(x_{i}\right) \mathrm{e}^{-\left(\lambda x_{i}\right)^{c}} \log ^{2}\left(\lambda x_{i}\right)}{\left[1-\varphi^{a}\left(x_{i}\right)\right]^{2}} \\
& \times\left\{\beta-\beta \varphi^{a}\left(x_{i}\right)-\left(\lambda x_{i}\right)^{c}+\left(\lambda x_{i}\right)^{c} \varphi^{a}\left(x_{i}\right)-a \Gamma^{-1}(\beta)\left(\lambda x_{i}\right)^{c \beta}\right. \\
& \times \varphi^{-1}\left(x_{i}\right) \mathrm{e}^{-\left(\lambda x_{i}\right)^{c}}+\Gamma^{-1}(\beta)\left(\lambda x_{i}\right)^{c \beta} \varphi^{-1}\left(x_{i}\right) \mathrm{e}^{-\left(\lambda x_{i}\right)^{c}} \\
& \left.-\Gamma^{-1}(\beta)\left(\lambda x_{i}\right)^{c \beta} \varphi^{a-1}\left(x_{i}\right) \mathrm{e}^{-\left(\lambda x_{i}\right)^{c}}\right\},
\end{aligned}
$$

$$
\begin{aligned}
U_{c, \beta}(\boldsymbol{\theta})= & n \log \lambda[1+2 c \beta \log \lambda]+\sum_{i=1}^{n} \log x_{i} \\
+ & \frac{a-1}{\Gamma(\beta)} \sum_{i=1}^{n} \frac{\left(\lambda x_{i}\right)^{c \beta} \mathrm{e}^{-\left(\lambda x_{i}\right)^{c}} \log \left(\lambda x_{i}\right)}{\varphi\left(x_{i}\right)} \\
\times & \left\{\psi(\beta)-\log \left(\lambda x_{i}\right)^{c}+\varphi^{-1}\left(x_{i}\right) \rho\left(x_{i}\right)\right\}+\frac{a(a-1)}{\Gamma(\beta)} \\
\times & \sum_{i=1}^{n} \frac{\left(\lambda x_{i}\right)^{c \beta} \varphi^{a-2}\left(x_{i}\right) \mathrm{e}^{-\left(\lambda x_{i}\right)^{c}} \log \left(\lambda x_{i}\right)}{1-\varphi^{a}\left(x_{i}\right)}\left\{\psi(\beta)+\varphi\left(x_{i}\right)\right. \\
\times & \left.\log \left(\lambda x_{i}\right)^{c}+\rho\left(x_{i}\right)\left\{1+a+a \varphi^{a}\left(x_{i}\right)\left[1-\varphi^{a}\left(x_{i}\right)\right]^{-1}\right\}\right\}, \\
U_{c, \lambda}(\boldsymbol{\theta})= & n \beta \lambda^{-1}-\lambda^{c-1}\left\{(c \log \lambda+1) \sum_{i=1}^{n} x_{i}^{c}+\sum_{i=1}^{n} x_{i}^{c} \log x_{i}^{c}\right\} \\
& \frac{a-1}{\Gamma(\beta)} \sum_{i=1}^{n} \frac{x_{i}\left(\lambda x_{i}\right)^{c \beta-1} \mathrm{e}^{-\left(\lambda x_{i}\right)^{c}}}{\varphi\left(x_{i}\right)}\left\{1+c \log \left(\lambda x_{i}\right)[\beta\right. \\
& \left.\left.\left(\lambda x_{i}\right)^{c}+\Gamma^{-1}(\beta)\left(\lambda x_{i}\right)^{c \beta} \varphi^{-1}\left(x_{i}\right) \mathrm{e}^{-\left(\lambda x_{i}\right)^{c}}\right]\right\}+a(b-1)
\end{aligned}
$$




$$
\begin{aligned}
& \times \sum_{i=1}^{n} \frac{x_{i}\left(\lambda x_{i}\right)^{c \beta-1} \varphi^{a}\left(x_{i}\right) \mathrm{e}^{-\left(\lambda x_{i}\right)^{c}} \log \left(\lambda x_{i}\right)}{\left[1-\varphi^{a}\left(x_{i}\right)\right]^{2}}\left\{-c \Gamma^{-1}(\beta)\right. \\
& \times\left(\lambda x_{i}\right)^{c \beta} \varphi^{-1}\left(x_{i}\right) \mathrm{e}^{-\left(\lambda x_{i}\right)^{c}}\left[1+a-\varphi^{a}\left(x_{i}\right)\right]+\left[1-\varphi^{a}\left(x_{i}\right)\right] \\
& \left.\times\left[c \beta-c\left(\lambda x_{i}\right)^{c}+\left(\lambda x_{i}\right)^{-c \beta} \log ^{-1}\left(\lambda x_{i}\right)\right]\right\}, \\
& U_{\beta, \beta}(\theta)=-n a \psi^{\prime}(\beta)+(a-1) \sum_{i=1}^{n}\left\{\frac{\eta\left(x_{i}\right)+2 \Gamma(\beta) \eta_{1} \varphi\left(x_{i}\right)}{\Gamma^{2}(\beta) \varphi^{2}\left(x_{i}\right)}\right. \\
& \left.-\frac{\Gamma(\beta) \eta\left(x_{i}\right) \log \left(\lambda x_{i}\right)^{c}\left[1-\varphi\left(x_{i}\right)\right]}{\Gamma^{2}(\beta) \varphi^{2}\left(x_{i}\right)}\right\} \\
& \text { - } a(b-1) \sum_{i=1}^{n} \frac{\varphi^{a-1}\left(x_{i}\right)}{1-\varphi^{a}\left(x_{i}\right)}\left\{-\varphi\left(x_{i}\right)\left[\psi^{2}(\beta)-\psi^{\prime}(\beta)\right]\right. \\
& -2 \Gamma^{-1}(\beta)\left[\eta_{1}\left(x_{i}\right)-\psi(\beta) \eta\left(x_{i}\right)\right]+\frac{\rho^{2}\left(x_{i}\right)}{\varphi\left(x_{i}\right)} \\
& \times\left[1+a-\frac{a \varphi^{a}\left(x_{i}\right)}{1-\varphi^{a}\left(x_{i}\right)}\right]+\log \left(\lambda x_{i}\right)^{c}\left\{-2 \varphi\left(x_{i}\right)\right. \\
& \left.\left.+\Gamma^{-1}(\beta)\left[2 \eta\left(x_{i}\right)+\Gamma(\beta) \varphi\left(x_{i}\right) \log \left(\lambda x_{i}\right)^{c}\right]\right\}\right\},
\end{aligned}
$$

where

$$
\eta_{1}\left(x_{i}\right)=G_{4,0}^{3,4}\left(\begin{array}{l|l}
\left(\lambda x_{i}\right)^{c} & \begin{array}{l}
1,1,1 \\
0,0,0, \beta
\end{array}
\end{array}\right),
$$

and $\psi^{\prime}(\cdot)$ is the first derivative of the digamma function,

$$
\begin{aligned}
U_{\beta, \beta}(\theta) & =n \lambda^{-1}\left\{c-\psi(\beta)[1-c]-c^{2} \log \lambda[1+\beta]\right\} \\
& +\frac{c(a-1)}{\Gamma^{2}(\beta)} \sum_{i=1}^{n} \frac{x_{i} \eta\left(x_{i}\right)\left(\lambda x_{i}\right)^{c \beta-1} \mathrm{e}^{-\left(\lambda x_{i}\right)^{c}}}{\varphi^{2}\left(x_{i}\right)} \\
& +\frac{a c(b-1)}{\Gamma(\beta)} \sum_{i=1}^{n} \frac{x_{i}\left(\lambda x_{i}\right)^{c \beta-1} \varphi^{a-1}\left(x_{i}\right) \mathrm{e}^{-\left(\lambda x_{i}\right)^{c}}}{1-\varphi^{a}\left(x_{i}\right)} \\
& \times\left\{\psi(\beta) \log \left(\lambda x_{i}\right)^{c}+\frac{\rho\left(x_{i}\right)-\psi(\beta)\left[1+\Gamma^{-1}(\beta)\right]\left[1-\varphi\left(x_{i}\right)\right]}{\varphi\left(x_{i}\right)}\right. \\
& \left.\times\left[-1+a+\frac{a \varphi^{a-1}\left(x_{i}\right)}{1-\varphi^{a}\left(x_{i}\right)}\right]\right\},
\end{aligned}
$$




$$
\begin{aligned}
U_{\lambda, \lambda}(\theta) & =n c \beta \lambda^{-2}\left[-c \beta+\lambda^{1-c \beta}(-1+c \beta)\right]-c(-1+c) \lambda^{c-2} \sum_{i=1}^{n} x_{i}^{c} \\
& +\frac{c(a-1)}{\Gamma(\beta)} \sum_{i=1}^{n} x_{i}^{2}\left(\lambda x_{i}\right)^{c \beta-2} \varphi^{-1}\left(x_{i}\right) \mathrm{e}^{-\left(\lambda x_{i}\right)^{c}}\left\{1-c \beta+c\left(\lambda x_{i}\right)^{c}\right. \\
& \left.-c \Gamma^{-1}(\beta)\left(\lambda x_{i}\right)^{c \beta} \varphi^{-1}\left(x_{i}\right) \mathrm{e}^{-\left(\lambda x_{i}\right)^{c}}\right\}+\frac{a c(b-1)}{\Gamma(\beta)} \\
& \times \sum_{i=1}^{n} \frac{x_{i}^{2}\left(\lambda x_{i}\right)^{c \beta-2} \varphi^{a-1}\left(x_{i}\right) \mathrm{e}^{-\left(\lambda x_{i}\right)^{2}}}{\left[1-\varphi^{a}\left(x_{i}\right)\right]^{2}}\left\{1-c \beta+c\left(\lambda x_{i}\right)^{c}\right. \\
& -\varphi^{a}\left(x_{i}\right)\left[1-c \beta+c\left(\lambda x_{i}\right)^{c}\right]+\left(\lambda x_{i}\right)^{c} \mathrm{e}^{-\left(\lambda x_{i}\right)^{c}} \\
& \left.\times\left[-1+a+\Gamma^{-1}(\beta) \varphi^{a-1}\left(x_{i}\right)\right]\right\} .
\end{aligned}
$$

\section{References}

[1] G. Chen, N. Balakrishnan, A general purpose approximate goodness-of-fit test, Journal of Quality Technology, 27 (1995), 154-161.

[2] G.M. Cordeiro, E.M.M. Ortega, G.O. Silva, The exponentiated generalized gamma distribution with application to lifetime data. Journal of Statistical Computation and Simulation, 81 (2013), 827-842.

[3] G.M. Cordeiro, D.C.C. Cunha, E.M.M. Ortega, The Exponentiated Generalized Class of Distributions, Journal of Data Science, 11 (2013), 777-803.

[4] D.R. Cox, D.V. Hinkley, Theoretical Statistics, Chapman and Hall, UK (1974).

[5] I.S. Gradshteyn, I.M. Ryzhik, Table of integrals, series, and products, Academic Press, USA (2007).

[6] R.C. Gupta, P.L. Gupta, R.D. Gupta, Modeling Failure Time Data by Lehman Alternatives, Communications in Statistics-Theory and Methods, 27 (1998), 887-904.

[7] R.D. Gupta, D. Kundu, Exponentiated exponential family: an alternative to gamma and Weibull distributions. Biometrical Journal, 43 (2001), 117130. 
[8] D.N.P. Murthy, M. Xie, R. Jiang, Weibull Models, John Wiley and Sons, USA (2004).

[9] G.S. Mudholkar, D.K. Srivastava, Exponentiated Weibull family for analyzing bathtub failure-rate data, IEEE Transactions on Reliability, 42 (1993), 299-302.

[10] G.S. Mudholkar, D.K. Srivastava, M. Friemer, The exponentiated Weibull family: A reanalysis of the bus-motor-failure data, Technometrics, 37 (1995), 436-445.

[11] S. Nadarajah, S. Kotz, The Exponetiated Type Distributions, Acta Applicandae Mathematicae, 92 (2006), 97-111.

[12] S. Nadarajah, A.K. Gupta, The exponentiated gamma distribution with application to drought data, Calcutta Statistical Association Bulletin, $\mathbf{5 9}$ (2007), 29-54.

[13] A.P. Prudnikov, Y.A. Brychkov, O.I. Marichev, Integrals and Series, volume 1, Gordon and Breach Science Publishers, Germany (1986).

[14] R Development Core Team, $R$ : A Language and Environment for Statistical Computing. R Foundation for Statistical Computing, Vienna (2012).

[15] A. Rényi, On measures of entropy and information. In: Proceedings of the 4th Berkeley Symposium on Mathematical Statistics and Probability, University of California Press: Berkeley, 01 (1961), 547-561.

[16] E.W. Stacy, A generalization of the gamma distribution, Annals of Mathematical Statistics, 33 (1962), 1187-1192.

[17] E.W. Stacy, G.A. Mihram, Parameter estimation for a generalized gamma distribution. Technometrics, 7 (1965), 349-358. 
